balance with amino acid plus glucose solution $(P=0.0063)$, indicating that nutritional state might influence the metabolic effects of the dialysis mixture.

Original article Tjiong HL et al. (2006) Peritoneal dialysis with solutions containing amino acids plus glucose promotes protein synthesis during oral feeding. Clin J Am Soc Nephrol 2: $74-80$

\section{Use of an abdominal band reduces postdialytic orthostatic hypotension in some patients}

Postdialytic orthostatic hypotension $(\mathrm{OH})$ is a risk factor for mortality in patients receiving hemodialysis. A study by Yamamoto et al. has concluded that abdominal compression with an inflatable band can be an effective means of ameliorating postdialytic $\mathrm{OH}$, especially in patients with stable systolic heart function. The band acts by compressing the abdominal vascular bed, thereby shifting the blood pool to the thoracic space.

The study evaluated 25 chronic hemodialysis patients with postdialytic $\mathrm{OH}$ for change in systolic blood pressure (SBP) between supine and standing positions. Patients rested in a supine position for $15 \mathrm{~min}$. Once their blood pressure was confirmed to have stabilized, they participated in an active standing test and their blood pressure was taken after $1 \mathrm{~min}$. Without use of the abdominal band, the change in SBP between supine and standing positions was significantly greater after a hemodialysis session than before $(-36.2 \mathrm{mmHg}$ vs $-13.1 \mathrm{mmHg}$; $P<0.0001)$. Using the inflatable band after dialysis significantly reduced the change in SBP compared with no band $(-19.4 \mathrm{mmHg}$ vs $-36.2 \mathrm{~mm} \mathrm{Hg} ; P<0.002)$. The band increased standing SBP, but did not elevate supine SBP; it also tended to reduce the occurrence of symptoms of hypotension.

The authors identified a bimodal response to the band, and designated a subset of eight patients 'responders' (change in SBP $\geq 25 \mathrm{mmHg}$ with the band). Responders had better ejection fractions, and lower atrial natriuretic peptide concentrations, than nonresponders $(P<0.02$ for both).

Original article Yamamoto N et al. (2006) Treatment of post-dialytic orthostatic hypotension with an inflatable abdominal band in hemodialysis patients. Kidney Int 70: 1793-1800

\section{Survival after cardiac arrest in HD clinic unimproved by access to external defibrillator}

Placement of automated external defibrillators (AEDs) in all outpatient dialysis centers has been recommended in order to improve outcomes from cardiac arrest. Researchers in the US have investigated whether provision of AEDs improves survival after cardiac arrest in patients undergoing hemodialysis (HD).

In total, 797 patients suffered a confirmed cardiac arrest in one of 565 clinics; 68 patients were excluded from the final analysis because of missing outcomes data or 'do not resuscitate' orders. Of the 729 remaining patients, 492 had cardiac arrests in a clinic without an AED and 237 had cardiac arrests in a clinic with an AED.

Unadjusted survival rates at 30 days and at 1 year were not significantly different between patients who experienced cardiac arrest in clinics with or without AEDs ( $19 \%$ vs $15 \%$ at 30 days, and $9.5 \%$ vs $7.8 \%$ at 1 year). The presence of an AED was not associated with improved survival in an unadjusted model (hazard ratio $0.91,95 \% \mathrm{Cl} 0.78-1.07 ; P=0.26$ ) or in a model adjusted for all potential confounders (hazard ratio $0.98,95 \% \mathrm{Cl} 0.82-1.18 ; P=0.83$ ). When the analysis was limited to those patients for whom AED use was confirmed, no improvement in survival with AED use was observed.

The authors conclude that the strategy of placing AEDs in HD clinics does not seem to improve the poor outcome from in-center cardiac arrest in patients on HD in the US.

Original article Lehrich RW et al. (2007) Automated external defibrillators and survival from cardiac arrest in the outpatient hemodialysis clinic. J Am Soc Nephrol 18: 312-320

\section{Cooled dialysate ameliorates hemodialysis-induced left ventricular dysfunction}

Several studies have reported development of subclinical myocardial ischemia in response to the stress of hemodialysis; repeated cumulative damage owing to subclinical ischemia can lead to heart failure. In a small study, Selby et al. demonstrated that by cooling the dialysate-and thus reducing the incidence and severity of intradialytic hypotension (IDH) - the likelihood of developing hemodialysis-induced 